\title{
Efecto de variantes de los genes ABCB1 y CYP3A4 sobre la respuesta terapéutica a atorvastatina en individuos chilenos hipercolesterolémicos
}

\author{
Alexy Rosales $Z^{1,2,3}$, Luis A. Salazar ${ }^{2,3}$ \\ 1 Programa de Doctorado en Ciencias, mención Biología Celular y Molecular Aplicada, \\ Universidad de La Frontera, Temuco, Chile \\ 2 Centro de Biología Molecular y Farmacogenética, Depto. de Ciencias Básicas, \\ Facultad de Medicina, Universidad de La Frontera, Temuco, Chile. \\ 3 Núcleo de Desarrollo Científico - Tecnológico en Biorecursos (BIOREN), \\ Universidad de La Frontera, Temuco, Chile. \\ Trabajo financiado por Fundación Araucaria \& Proyecto DIUFRO DI09-1007, \\ Dirección de Investigación y Desarrollo, Universidad de La Frontera.
}

\section{Resumen:}

Introducción: El tratamiento farmacológico con estatinas es eficaz en el control de la hipercolesterolemia. Sin embargo, su respuesta terapéutica presenta gran variabilidad interindividual. Factores como edad, género y etnia han sido relacionados a esta variabilidad. Además, estudios recientes demuestran que polimorfismos genéticos también pueden influir sobre la respuesta terapéutica a estos hipolipemiantes. Así, el objetivo del presente trabajo fue investigar el efecto de tres polimorfismos de los genes ABCB1 (3435C $>$ T y 2677G $>A>T$ ) y CYP3A4 (-290A>G) sobre la respuesta al tratamiento con atorvastatina en individuos chilenos hipercolesterolémicos.

Métodos: Fueron evaluados 94 individuos hipercolesterolémicos adultos, 35 hombres y 59 mujeres, tratados con atorvastatina (10mg/día) durante 4 semanas. Se realizó medición de los niveles séricos de colesterol total, triglicéridos, HDL-c y LDL-c, antes y después del tratamiento. La genotipificación de los polimorfismos se realizó mediante PCR-RFLP.

Resultados: Después de 4 semanas de tratamiento, se observaron reducciones significativas en los niveles de colesterol total $(275 \pm 18$ vs. $235 \pm 22 \mathrm{mg} / \mathrm{dL}, \mathrm{p}<0.001)$, LDL-c $(183 \pm 16$ vs. $146 \pm 24 \mathrm{mg} / \mathrm{dL}, \mathrm{p}<0.001)$ y triglicéridos ( $225 \pm 48$ vs. $172 \pm 51 \mathrm{mg} / \mathrm{dL}, \mathrm{p}<0.001)$. Además, se observó un aumento en las concentraciones de HDL-c $(47 \pm 10$ vs. $55 \pm 7, \mathrm{p}<0.001)$. La distribución de genotipos para el polimorfismo $3435 \mathrm{C}>\mathrm{T}$ (ABCB1) fue: $\mathrm{CC}=47.9 \%, \mathrm{CT}=41.5 \%$ y $\mathrm{TT}=10.6 \%$; para el polimorfismo trialélico $2677 \mathrm{G}>\mathrm{A}>\mathrm{T}$ ( $\mathrm{ABCB} 1$ ): $\mathrm{AA}=1.1 \%$, $\mathrm{GA}=14.9 \%, \mathrm{GT}=28.7 \%, \mathrm{TA}=9.6 \%, \mathrm{TT}=8.5 \% \quad \mathrm{y}$ $\mathrm{GG}=37.2 \%$ y para $-290 \mathrm{~A}>\mathrm{G}$ (CYP3A4) fue: $\mathrm{AA}=$ $77.7 \%, \mathrm{AG}=22.3 \%$ y $\mathrm{GG}=0.0 \%$. Los portadores del alelo $\mathrm{G}$ de la variante $-290 \mathrm{~A}>\mathrm{G}(\mathrm{CYP} 3 \mathrm{~A} 4)$ presentaron mayor reducción de colesterol total $(\mathrm{p}=0.001)$ y LDL-C $(\mathrm{p}<0.001)$, y un mayor aumento de HDL-c $(\mathrm{p}<0.001)$. No se observaron diferencias significativas para los otros dos polimorfismos estudiados.

Conclusión: La respuesta terapéutica a atorvastatina (10mg/día/1mes) es influenciada por la variante -290A>G del gen CYP3A4 en la población estudiada.

\section{Correspondencia:}

Dr. Luis Antonio Salazar

Centro de Biología Molecular y Farmacogenética

Departamento de Ciencias Básicas,

Facultad de Medicina,

Universidad de La Frontera.

Av. Francisco Salazar 01145, Casilla 54-D, Temuco

Fono: (45) 592895 - Fax: (45) 592832.

Correo Electrónico: Isalazar@ufro.cl 


\section{Effect of ABCB1 and CYP3A4 gene polymorphisms on the therapeutic response to atorvastatin in Chilean hypercholesterolemic individuals}

Background: : statins are effective in the control of hypercholesterolemia. However, the therapeutic response to these drugs presents a great inter-individual variability. Factors such as age, gender and ethnicity have been associated to this variability. Recent studies show that polymorphisms can influence the lipid-lowering response to statins. The aim of the present study was to investigate the effect of three polymorphisms $3435 \mathrm{C}>\mathrm{T}$ (ABCB1), 2677G $>\mathrm{A} / \mathrm{T}$ (ABCB1) and -290 $\mathrm{A}>\mathrm{G}$ (CYP3A4) gene on the response to atorvastatin treatment in Chilean hypercholesterolemic individuals. Methods: 94 hypercholesterolemic individuals, were treated with atorvastatin, $10 \mathrm{mg} /$ day for 4 weeks. We determined serum levels of total cholesterol, HDLc, LDL-c and triglycerides, before and after treatment. The polymorphisms were analyzed by PCR-RFLP. Results: Significant reductions were observed in total cholesterol ( $275 \pm 18$ vs. $235 \pm 22 \mathrm{mg} / \mathrm{dL}, \mathrm{p}<0.001)$, LDL-c $(183 \pm 16$ vs. $146 \pm 24 \mathrm{mg} / \mathrm{dL}, \mathrm{p}<0.001)$ and triglycerides levels $(225 \pm 48$ vs. $172 \pm 51 \mathrm{mg} / \mathrm{dL}$,

\section{Introducción:}

Una de las principales causas de muerte a nivel mundial, en ambos sexos, son aquellas vinculadas al aparato circulatorio, en las cuales las alteraciones del metabolismo lipídico son, sin lugar a dudas, el factor de riesgo más importante para el desarrollo de estas, demostrado así la estrecha relación existente entre las concentraciones de colesterol plasmático y enfermedad cardiovascular ${ }^{1}$. Según datos epidemiológicos de nuestro país (ENS 2003) ${ }^{2}$, un 55 por ciento de la población tiene riesgo de padecer este tipo de enfermedad y más de un tercio de sus habitantes poseen niveles de colesterol elevado, lo cual sumado a otras patologías crónicas como sobrepeso, obesidad e hipertensión arterial, las cuales afectan en promedio a un $30 \%$ de la población, y los consiguientes factores de riesgo asociados como tabaquismo, alcohol y sedentarismo, convierten a los chilenos, en edades cada vez más tempranas, en un blanco perfecto de accidentes cardiovasculares $^{1-3}$.

Una de las alternativas para ayudar a disminuir las altas tasas de morbilidad y mortalidad por enfermedades cardiovasculares, es tratar farmacológicamente a los $\mathrm{p}<0.001)$. In addition, an increase in the HDL-C levels was observed $(47 \pm 10$ vs. $55 \pm 7, \mathrm{p}<0.001)$. The genotype distribution for $3535 \mathrm{C}>\mathrm{T}$ variant of $\mathrm{ABCB} 1$ gene was: $\mathrm{CC}=47.9 \%, \mathrm{CT}=41.5 \%$ and $\mathrm{TT}=10.6 \%$. The genotype distribution for $2677 \mathrm{G}>\mathrm{A} / \mathrm{T}$ polymorphism (ABCB1) was $\mathrm{AA}=1.1 \%, \mathrm{GA}=14.9 \%, \mathrm{GT}=28.7 \%$, $\mathrm{TA}=9.6 \%, \mathrm{TT}=8.5 \%$ and $\mathrm{GG}=37.2 \%$. The genotype distribution for $-290 \mathrm{~A}>\mathrm{G}$ variant of CYP3A4 gene was: $\mathrm{AA}=77.7 \%$. $\mathrm{AG}=22.3 \%$ and $\mathrm{GG}=0.0 \%$. Subjects carrying the $\mathrm{G}$ allele for the $-290 \mathrm{~A}>\mathrm{G}$ variant of the CYP3A4 gene, exhibited a greater reduction in total cholesterol $(p=0.001)$ and LDL-C levels $(p<0.001)$. No differences were observed for the other polymorphisms studied.

Conclusion: This study suggests that the therapeutic response to atorvastatin $(10 \mathrm{mg} /$ day during 4 weeks) is influenced by the $-290 \mathrm{~A}>\mathrm{G}$ variant of CYP3A4 gene.

Keywords: Polymorphisms; CYP3A4; atorvastatin; $\mathrm{ABCB} 1$

pacientes que presenten niveles elevados de colesterol, los cuales demuestran una significativa disminución de estas tasas, al ser tratados con inhibidores de la 3-hidroxi-3-metilglutaril coenzima A (HMG-CoA) reductasa o estatinas, terapia farmacológica más utilizada, por su demostrada eficacia para reducir el colesterol sérico con el mínimo de efectos clínicos adversos ${ }^{4}$.

Su mecanismo de acción consiste en inhibir de forma competitiva y reversible la enzima HMG-CoA reductasa que cataliza la biosíntesis de colesterol en el hígado, al bloquear la formación de ácido mevalónico y disminuir la síntesis intracelular de colesterol total; lo que resulta en un aumento compensatorio en la expresión de receptores para LDL en las células hepáticas, disminuyendo los niveles de LDL-c circulante y colesterol total (hasta un 50\%); asimismo, originan un modesto descenso de los niveles de triglicéridos (TG) y un ligero aumento de los niveles de HDL-c ${ }^{5,6}$. Además, junto a los resultados exitosos en el manejo de las hipercolesterolemia, las estatinas han demostrado otros efectos benéficos conocidos como efectos "pleiotrópicos", entre ellos podemos destacar, su acción antiinflamatoria, anti-trombótica, anti-proliferativa y antioxidante $^{7,8}$. 
La prescripción de estatinas es amplia y se establece como terapia de primera línea, tanto en prevención primaria como secundaria. Sin embargo, el beneficio del tratamiento entre pacientes varía considerablemente, y estaría determinado, entre otros factores, por variaciones genéticas ${ }^{9}$. A la fecha, se han investigado más de 40 genes que afectan la respuesta a estatinas ${ }^{10}$, relacionados tanto con la farmacocinética (enzimas de metabolización y proteínas de transporte) como con la farmacodinámica del medicamento (receptores y vías de transducción de señal) ${ }^{11}$. Estas variaciones incluyen a genes que actúan en la absorción intestinal de colesterol como la apolipoproteína E (APOE), transportador de ATP (ABC); la producción de colesterol, como la HMG-CoA reductasa; el metabolismo de las lipoproteínas como la apolipoproteína $\mathrm{B}$ y el receptor de LDL, como además, de aquellos que actúan en la vía de la citocromo P450 ${ }^{12-15}$.

Las estatinas son metabolizadas por vía hepática, principalmente por la familia de la citocromo P450. La isoforma CYP3A4 es la más importante de esta familia de enzimas. Kajinami et al. ${ }^{16}$, en un estudio que evaluó 340 pacientes hipercolesterolémicos, demostraron que polimorfismos del gen CYP3A4 influyen en la respuesta terapéutica a atorvastatina. Además, variantes genéticas asociadas a proteínas transportadoras, ABCB1 o MDR1 (proteína de resistencia a múltiples drogas), que se expresan tanto en hígado, intestino, y riñón; y que serían las primeras en interactuar con el medicamento, también han sido relacionadas con la respuesta a estos fármacos ${ }^{17}$. Se ha asociado al polimorfismo trialélico G2677A/T del gen ABCB1 con una reducción en los niveles de LDL-colesterol, al ser tratados con el medicamento pravastatina ${ }^{18}$.

Así, el objetivo del presente estudio fue investigar el efecto de tres variantes genéticas sobre la respuesta terapéutica al hipolipemiante atorvastatina $(10 \mathrm{mg} / \mathrm{día} / 1$ mes) en individuos chilenos hipercolesterolémicos.

\section{Métodos:}

\section{Sujetos}

Se analizó un total de 94 individuos adultos (35 hombres y 59 mujeres), no relacionados, cuyo rango de edad fluctuó entre 33 y 74 años. La condición clínica para la selección de los participantes en este estudio fue el diagnóstico de hipercolesterolemia primaria (Colesterol total $\geq 240 \mathrm{mg} / \mathrm{dL}$ ) y niveles de LDL-c $\geq$ $190 \mathrm{mg} / \mathrm{dL}$, si tenían entre 0 y 1 factor de riesgo; $\geq 160$ $\mathrm{mg} / \mathrm{dL}$, si tenían 2 o más factores de riesgo o $\geq 100$ $\mathrm{mg} / \mathrm{dL}$ si tenían enfermedad coronaria conocida, de acuerdo a las recomendaciones del National Cholesterol Education Program (NCEP) ${ }^{19}$ y que requerían tratamiento farmacológico, debido a que no respondieron a intervención dietaria, indicada por un período previo de 3 meses; todos pertenecientes al Centro de Salud Municipal Federico Thieme, Comuna de Perquenco, Región de La Araucanía.

Los criterios de exclusión del estudio fueron los siguientes: Diabetes mellitus, hipotiroidismo $(\mathrm{TSH}>$ 7,5 $\mathrm{mU} / \mathrm{L}$ ), enfermedades hepáticas, enfermedades renales, medicación hipolipemiante al momento de la evaluación, uso de contraceptivos orales o anabolizantes y embarazo. Individuos con terapia concomitante con fibratos y mibefradil (bloqueador de los canales de calcio), también fueron excluidos. A los pacientes que consintieron participar del estudio, se les aplicó un cuestionario sobre historia familiar de enfermedad cardiovascular y antecedentes mórbidos personales.

Los pacientes seleccionados recibieron como tratamiento farmacológico $10 \mathrm{mg}$ /día de atorvastatina por el lapso de un mes. Al término de este período se tomó una nueva muestra de sangre para evaluar sus niveles de lípidos. La adherencia del paciente al tratamiento se verificó por el recuento de los comprimidos no ingeridos al final del mes.

El estudio contó con la aprobación del Comité de Ética Científica del Servicio de Salud Araucanía Sur (Región de La Araucanía, Chile). Todos los participantes firmaron voluntariamente un consentimiento informado.

\section{Determinaciones bioquímicas y antropométricas}

Las muestras de sangre fueron obtenidas por venopunción periférica, según técnicas de enfermería habituales, con un ayuno mínimo de $12 \mathrm{~h}$, por medio del sistema de extracción al vacio, tomando dos muestras: una muestra con anticoagulante EDTA $(1 \mathrm{mg} / \mathrm{mL}$ de sangre), para la extracción de material genético (DNA) y otra sin anticoagulante para la obtención de suero, en el cual se realizó las determinaciones bioquímicas. Las muestras fueron tomadas en el mismo Centro Asistencial por personal calificado y transportadas al laboratorio, en cadena de frío $\left(4^{\circ} \mathrm{C}\right)$. Las muestras de sangre sin anticoagulante fueron centrifugadas a $2500 \mathrm{rpm}$ (1050 g) por 10 minutos para obtener el suero utilizado en las determinaciones bioquímicas.

Se realizó la medición de colesterol total y triglicéridos por los métodos enzimáticos-colorimétricos CHOD-PAP y GPO-PAP, respectivamente ${ }^{20,21}$, en un analizador convencional. El colesterol de las HDL-C se midió previa precipitación selectiva de las LDL y VLDL con ácido fosfotúngstico e iones magnesio por 


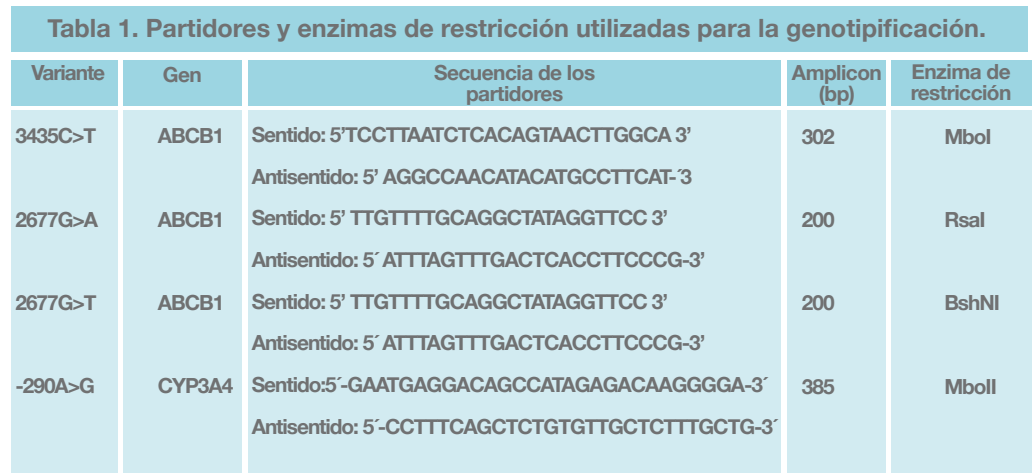

el método CHOD-PAP ${ }^{22}$. La concentración de colesterol de las LDL (LDL-C) fue calculada mediante la fórmula de Friedewald et al. ${ }^{23}$, cuando la concentración de triglicéridos fue inferior a $400 \mathrm{mg} / \mathrm{dL}$.

Los niveles séricos de glucosa, ácido úrico y creatinina se determinaron mediante métodos enzimáticocolorimétricos. La seguridad clínica del tratamiento fue evaluada buscando síntomas de efectos adversos y mediante las determinaciones de actividad sérica de las enzimas ALT, AST, $\gamma$-GT y CK. Además, se realizaron mediciones antropométricas de peso, talla e IMC.

\section{Análisis Molecular}

El ADN genómico fue extraído de muestras de sangre total anticoaguladas con EDTA $(1 \mathrm{mg} / \mathrm{dL})$, mediante el método de precipitación salina modificado, descrito por Salazar et al. ${ }^{24}$. El estudio de las variantes 3435C > T (exón ${ }^{26}$, ABCB1), 2677G>A/T (exón 21, ABCB1) y -290A > G (región promotora, CYP3A4) se realizó mediante la técnica de reacción en cadena de la polimerasa (PCR), seguida de restricción enzimática con endonucleasas específicas, utilizando los partidores y condiciones descritas por Cascorbi et al. ${ }^{25}$, Rodrigues et al. ${ }^{26}$ y Cavalli et al. ${ }^{27}$, respectivamente (Tabla 1).

\section{Control de calidad de las determinaciones bioquímicas y moleculares}

La exactitud de las determinaciones bioquímicas fue controlada mediante la utilización de un control de calidad interno y un control de calidad externo, Prevecal, con sueros comerciales normales y patológicos (CEMES-BioSystems). La posibilidad de contaminación en los análisis moleculares, fue excluida por la utilización de control de reactivos en cada serie de amplificación. La correcta genotipificación de los polimorfismos estudiados fue confirmada mediante la repetición al azar del 10\% de los análisis previamente realizados y la utilización de muestras genotipificadas previamente para estos polimorfismos, donadas por la Dra. Rosario Hirata, del Laboratorio de Biología Molecular Aplicada al Diagnóstico de la Facultad de Ciencias Farmacéuticas de la Universidad de Sao Paulo (Brasil).

\section{Análisis estadístico}

El análisis de los datos obtenidos se realizó utilizando el Programa SigmaPlot 2008, Systat Software Inc., para Windows, versión 11.0 (San José, California, EE.UU.). Se comenzó analizando todas las variables descriptivamente. Se calcularon promedios, desviaciones standard, valores máximos y mínimos.

La asociación entre las diferentes variables analizadas fue comprobada mediante los test " $t$ " de Student pareado o ANOVA. Además, se utilizó el test de Chicuadrado $(\chi 2)$ para la comparación de proporciones y evaluar el cumplimiento del equilibrio de Hardy-Weinberg. El nivel de significancia estadístico considerado en este estudio fue $\mathrm{P}<0.05$.

\section{Resultados:}

En la Tabla 2, podemos observar las características biodemográficas de los sujetos estudiados. Se evaluaron las concentraciones séricas de lípidos, lipoproteínas, glucosa, creatinina y ácido úrico. En esta tabla se puede observar también, que según los niveles basales de colesterol total y LDL-colesterol, todos los individuos reclutados requerían tratamiento farmacológico. En relación al tratamiento, se observó una adherencia de $100 \%$ en los 94 sujetos investigados. No se observaron reacciones adversas.

Las concentraciones séricas de lípidos y lipoproteínas, en condiciones basales y post-tratamiento con el hipolipemiante atorvastatina (10mg/día/ $1 \mathrm{mes})$, se resumen en la Tabla 3. El nivel de reducción de colesterol total, LDL-c y triglicéridos fue significativo al término del tratamiento ( $\mathrm{p}<0.001$ ). Además, el fárma- 


\begin{tabular}{ll}
\hline \multicolumn{1}{|c|}{$\begin{array}{c}\text { Tabla 2. Características biodemográficas de los } \\
\text { individuos estudiados. }\end{array}$} \\
\hline Parámetros & $\mathrm{N}=94$ \\
\hline Edad, años & $58,9 \pm 11,1$ \\
Hombres/Mujeres, $\mathrm{n}$ & $35 / 59$ \\
IMC, Kg/m2 & $25,4 \pm 2,6$ \\
Glucosa, mg/dL & $97,5 \pm 9,9$ \\
Creatinina, $\mathrm{mg} / \mathrm{dL}$ & $0,98 \pm 0,2$ \\
Acido úrico, $\mathrm{mg} / \mathrm{dL}$ & $5,4 \pm 1,5$ \\
Colesterol total, $\mathrm{mg} / \mathrm{dL}$ & $275 \pm 18$ \\
HDL-c, $\mathrm{mg} / \mathrm{dL}$ & $47 \pm 10$ \\
LDL-c, $\mathrm{mg} / \mathrm{dL}$ & $183 \pm 16$ \\
Triglicéridos, $\mathrm{mg} / \mathrm{dL}$ & $225 \pm 48$ \\
\hline
\end{tabular}

$I M C$, indice de masa corporal; $L D L-c$, colesterol de lipoproteínas de baja densidad; HDL-c, Colesterol de lipoproteínas de alta densidad.

co produjo un aumento significativo de los niveles de HLD-c $(\mathrm{p}<0.001)$.

Las Tablas 4, 5 y 6 resumen las distribuciones genotípicas y frecuencias relativas de alelos para las tres variantes genéticas investigadas. Para la variante -290 A $>\mathrm{G}$ del gen CYP3A4 (Tabla 4), la distribución de los genotipos fueron las siguientes: $\mathrm{AA}=77.7 \%$ y AG= $22.3 \%$; el genotipo mutado GG no fue observado en la población en estudio. La frecuencia para el alelo mutado -290G fue 0.112. En relación al polimorfismo $3435 \mathrm{C}>\mathrm{T}$ del gen $\mathrm{ABCB} 1$, la distribución de genotipos fue: $\mathrm{CC}=47.9 \%$; $\mathrm{CT}=41.5 \%$ y TT $=10.6 \%$, y la frecuencia del alelo mutado $3435 \mathrm{~T}$ fue 0.313. Para el polimorfismo trialélico $2677 \mathrm{G}>\mathrm{A} / \mathrm{T}$ del gen $\mathrm{ABCB} 1$ (Tabla 6), la distribución genotípica encontrada fue la siguiente: $\mathrm{AA}=1 \% ; \mathrm{GA}=14.9 \% ; \mathrm{GT}=28.7 \%, \mathrm{TA}=$ 9.6\%; $\mathrm{TT}=8.5 \%$ y $\mathrm{GG}=37.2 \%$. Las frecuencias de los alelos mutados $2677 \mathrm{~T}$ y $2677 \mathrm{~A}$ fueron 0.277 y 0.113 , respectivamente. La distribución genotípica para las tres variantes investigadas fue concordante con el equilibrio Hardy-Weinberg ( $>00.005)$.

Al comparar la respuesta terapéutica a la atorvastatina, según los tres polimorfismos investigados (Tablas

\begin{tabular}{|c|c|c|c|}
\hline Parámetros & Basal & Post-tratamiento & $\mathbf{P}^{*}$ \\
\hline CT, mg/dL & $275 \pm 18$ & $235 \pm 22$ & $<0.001$ \\
\hline HDL-c, mg/dL & $47 \pm 10$ & $55 \pm 6$ & $<0.001$ \\
\hline LDL-c, mg/dL & $183 \pm 16$ & $146 \pm 24$ & $<0.001$ \\
\hline Triglicéridos, mg/dL & $225 \pm 48$ & $172 \pm 51$ & $<0.001$ \\
\hline
\end{tabular}

$C T$, colesterol total; $L D L$-c, colesterol de lipoproteínas de baja densidad; HDL-c, Colesterol de lipoproteínas de alta densidad.

* Test t de Student pareado.

\begin{tabular}{|c|c|c|c|c|c|}
\hline \multicolumn{2}{|c|}{$\begin{array}{l}\text { Tabla 4. Distribución de genotipos y frecuencia relativa de } \\
\text { alelos para el polimorfismo -290 A>G del gen CYP3A4 en } \\
\text { individuos chilenos hipercolesterolémicos. }\end{array}$} & & & & \\
\hline POLIMORFISMO & & & & \multicolumn{2}{|c|}{ ALELOS } \\
\hline \multirow[t]{3}{*}{$-290 A>G$} & AA & AG & GG & A & G \\
\hline & 77.7 & 22.3 & 0.0 & & \\
\hline & (73) & (21) & (0) & 0.888 & 0.112 \\
\hline
\end{tabular}

Número de individuos en paréntesis.

Equilibrio de Hardy Weinberg: $\chi 2=1.48 ; P=0.22$

Tabla 5. Distribución de genotipos y frecuencia relativa de alelos para el polimorfismo $3435 \mathrm{C}>\mathrm{T}$ del gen ABCB1 (MDR1) en individuos hipercolesterolémicos chilenos.

\begin{tabular}{|l|lcc|c|c|}
\hline POLIMORFISMO & \multicolumn{3}{|c|}{ GENOTIPOS, \% } & \multicolumn{3}{|c|}{ ALELOS } \\
\hline 3435 C $>$ T & CC & CT & T & C & T \\
& 47.9 & 41.5 & 10.6 & & \\
& (45) & (39) & (10) & 0.686 & 0.313 \\
\hline
\end{tabular}

Número de individuos en paréntesis.

Equilibrio de Hardy Weinberg: $\chi 2=0.12 ; P=0.72$

7, 8 y 9), podemos observar que sólo el polimorfismo -290 A $>$ G del gen CYP3A4 afecta la respuesta al fármaco. Individuos con el genotipo heterocigoto mutado AG presentan mayor reducción de los niveles séricos de colesterol total y LDL colesterol, y un mayor aumento de los niveles de HDL colesterol $(\mathrm{p}<0.001)$.

\begin{tabular}{|c|c|c|c|c|c|c|c|c|c|}
\hline POLIMORFISMO & & \multicolumn{5}{|c|}{ GENOTIPOS,\% } & \multicolumn{3}{|c|}{ ALELOS } \\
\hline \multirow[t]{3}{*}{ G 2677 T/A } & GG & GT & $\pi$ & GA & TA & AA & G & $\begin{array}{lll}T & \end{array}$ & A \\
\hline & 37.2 & 28.7 & 8.5 & 14.9 & 9.6 & 1.1 & & & \\
\hline & (35) & (27) & (8) & (14) & (9) & (1) & 0.590 & 0.277 & 0.133 \\
\hline
\end{tabular}

Número de individuos en paréntesis. Equilibrio de Hardy Weinberg: $\chi 2=1.62, P=0.66$ 
Tabla 7. Concentraciones séricas de lípidos, antes y después, de sujetos tratados con atorvastatina $(10 / \mathrm{mg} /$ día/1mes), según genotipos para el polimorfismo -290 A>G del gen CYP3A4.

\begin{tabular}{|c|c|c|c|}
\hline Parámetros & $\begin{array}{c}\text { AA } \\
(n=73)\end{array}$ & $\begin{array}{c}A G \\
(n=21)\end{array}$ & $P^{*}$ \\
\hline \multicolumn{4}{|l|}{$\mathrm{CT}, \mathrm{mg} / \mathrm{dL}$} \\
\hline Basal & $273 \pm 16$ & $283.1 \pm 21$ & 0.018 \\
\hline Post-tratamiento & $238 \pm 21$ & $224.9 \pm 23$ & 0.019 \\
\hline Variación, \% & $-13 \pm 9$ & $-20 \pm 11$ & $<0.001$ \\
\hline \multicolumn{4}{|l|}{ HDL-c, mg/dL } \\
\hline Basal & $48 \pm 10$ & $44.7 \pm 8$ & 0.164 \\
\hline Post-tratamiento & $53 \pm 7$ & $56.4 \pm 5$ & 0.243 \\
\hline Variación, \% & $+16 \pm 13$ & $+29 \pm 18$ & $<0.001$ \\
\hline \multicolumn{4}{|l|}{ LDL-c, mg/dL } \\
\hline Basal & $181 \pm 16.3$ & $189 \pm 15$ & 0.035 \\
\hline Post-tratamiento & $150 \pm 22.1$ & $132 \pm 26$ & 0.002 \\
\hline Variación, \% & $-17 \pm 12.5$ & $-30 \pm 16$ & $<0.001$ \\
\hline \multicolumn{4}{|l|}{$\mathrm{TG}, \mathrm{mg} / \mathrm{dL}$} \\
\hline Basal & $219 \pm 42.8$ & $245 \pm 59$ & 0.026 \\
\hline Post-tratamiento & $169 \pm 51.9$ & $184 \pm 48$ & 0.231 \\
\hline Variación, \% & $-23 \pm 20.2$ & $-24 \pm 19$ & 0.852 \\
\hline
\end{tabular}

$C T$, colesterol total; $L D L-c$, colesterol de lipoproteínas de baja densidad; HDL-c, Colesterol de lipoproteínas de alta densidad; TG,

triglicéridos; * Test t de Student pareado.

Tabla 9. Concentraciones séricas de lípidos, antes y después, de sujetos tratados con atorvastatina $(10 \mathrm{mg}$ día/1mes), según genotipos, silvestre (GG) y mutados (No GG), para el polimorfismo trialélico 2677 G>A/T del gen ABCB1.

\begin{tabular}{|c|c|c|c|}
\hline Parámetros & $\underset{\substack{G=35) \\
(n=6}}{G}$ & $\begin{array}{l}\text { No GG } \\
(n=59)\end{array}$ & $\mathbf{P}^{*}$ \\
\hline \multicolumn{4}{|l|}{ CT, mg/dL } \\
\hline Basal & $278 \pm 19$ & $273 \pm 18$ & 0.270 \\
\hline Post-tratamiento & $233 \pm 23$ & $236 \pm 22$ & 0.456 \\
\hline Variación, \% & $-16 \pm 10$ & $-13 \pm 9$ & 0.210 \\
\hline \multicolumn{4}{|l|}{ HDL-c, mg/dL } \\
\hline Basal & $48 \pm 9$ & $47 \pm 10$ & 0.784 \\
\hline Post-tratamiento & $56 \pm 6$ & $55 \pm 7$ & 0.407 \\
\hline Variación, \% & $+19 \pm 18$ & $+18 \pm 14$ & 0.679 \\
\hline \multicolumn{4}{|l|}{ LDL-c, mg/dL } \\
\hline Basal & $184 \pm 16$ & $182 \pm 17$ & 0.534 \\
\hline Post-tratamiento & $141 \pm 25$ & $148 \pm 24$ & 0.199 \\
\hline Variación, \% & $-23 \pm 14$ & $-18 \pm 14$ & 0.128 \\
\hline \multicolumn{4}{|l|}{$\mathrm{TG}, \mathrm{mg} / \mathrm{dL}$} \\
\hline Basal & $230 \pm 52$ & $222 \pm 46$ & 0.422 \\
\hline Post-tratamiento & $177 \pm 48$ & $169 \pm 53$ & 0.472 \\
\hline Variación, \% & $-22 \pm 18$ & $-23 \pm 21$ & 0.868 \\
\hline
\end{tabular}

$C T$, colesterol total; $L D L-c$, colesterol de lipoproteínas de baja densidad; HDL-c, Colesterol de lipoproteínas de alta densidad; TG, triglicéridos. *Test t de Student pareado.
Tabla 8. Concentraciones séricas de lípidos, antes y después, de sujetos tratados con atorvastatina $(10 / \mathrm{mg} /$ día/1mes), según genotipos para el polimorfismo 3435 C>T del gen ABCB1.

\begin{tabular}{|c|c|c|c|c|}
\hline Parámetros & $\begin{array}{c}C C \\
(n=45)\end{array}$ & $\begin{array}{c}\text { CT } \\
(n=39)\end{array}$ & $\underset{(n=10)}{T}$ & $\mathbf{P}^{*}$ \\
\hline \multicolumn{5}{|l|}{ CT, mg/dL } \\
\hline Basal & $274 \pm 19$ & $275 \pm 17$ & $280 \pm 17$ & 0.646 \\
\hline Post-tratamiento & $232 \pm 24$ & $236 \pm 22$ & $242 \pm 25$ & 0.408 \\
\hline Variación, \% & $-15 \pm 9$ & $-14 \pm 9$ & $-13 \pm 11$ & 0.834 \\
\hline \multicolumn{5}{|l|}{ HDL-c, mg/dL } \\
\hline Basal & $46 \pm 9$ & $48 \pm 11$ & $47 \pm 9$ & 0.683 \\
\hline Post-tratamiento & $54 \pm 6$ & $56 \pm 7$ & $55 \pm 5$ & 0.434 \\
\hline Variación, \% & $+23 \pm 17$ & $+18 \pm 15$ & $+19 \pm 17$ & 0.421 \\
\hline \multicolumn{5}{|l|}{ LDL-c, mg/dL } \\
\hline Basal & $183 \pm 17$ & $182 \pm 17$ & $186 \pm 15$ & 0.820 \\
\hline Post-tratamiento & $143 \pm 23$ & $147 \pm 24$ & $150 \pm 29$ & 0.642 \\
\hline Variación, \% & $-21 \pm 13$ & $-19 \pm 15$ & $-18 \pm 19$ & 0.716 \\
\hline \multicolumn{5}{|l|}{$\mathrm{TG}, \mathrm{mg} / \mathrm{dL}$} \\
\hline Basal & $223 \pm 47$ & $224 \pm 49$ & $235 \pm 47$ & 0.783 \\
\hline Post-tratamiento & $175 \pm 50$ & $166 \pm 52$ & $183 \pm 56$ & 0.601 \\
\hline Variación, \% & $-22 \pm 17$ & $-24 \pm 23$ & $-23 \pm 17$ & 0.873 \\
\hline
\end{tabular}

$C T$, colesterol total; $L D L-C$, colesterol de lipoproteínas de baja densidad; HDL $-C$, Colesterol de lipoproteínas de alta densidad; $T G$, triglicéridos. *Test de ANOVA.

\section{Discusión:}

Las enfermedades cardiovasculares (ECV) son consideradas uno de los mayores problemas de nuestra década, aduciendo que cada año mueren más personas por esta causa que por alguna otra patología en el mundo, calculando que para el año 2015 podrían morir cerca de 20 millones de personas, pues se estima que siga siendo la principal causa de muerte, todo esto amparado en la creciente prevalencia de los factores de riesgo cardiovascular y el estancamiento de la reducción de muertes por esta causa, sugiriendo que la disminución continua de la tasa de mortalidad cardiovascular, observada en los últimas décadas, podría estar llegando a su fin ${ }^{28}$.

Ante estas alarmantes noticias, donde las campañas de prevención no están disminuyendo por sí solas estos factores de riesgo, la utilización de tratamiento farmacológico sigue siendo la alternativa de elección ante estas patologías. Pero la diversidad de respuesta presentada por los individuos, respondiendo de manera diferente ante el mismo medicamento, inclusive a las mismas dosis, han llevado a clínicos e investigadores a buscar nuevas alternativas para el éxito terapéutico.

El estudio de variantes genéticas en medicina ha genera- 


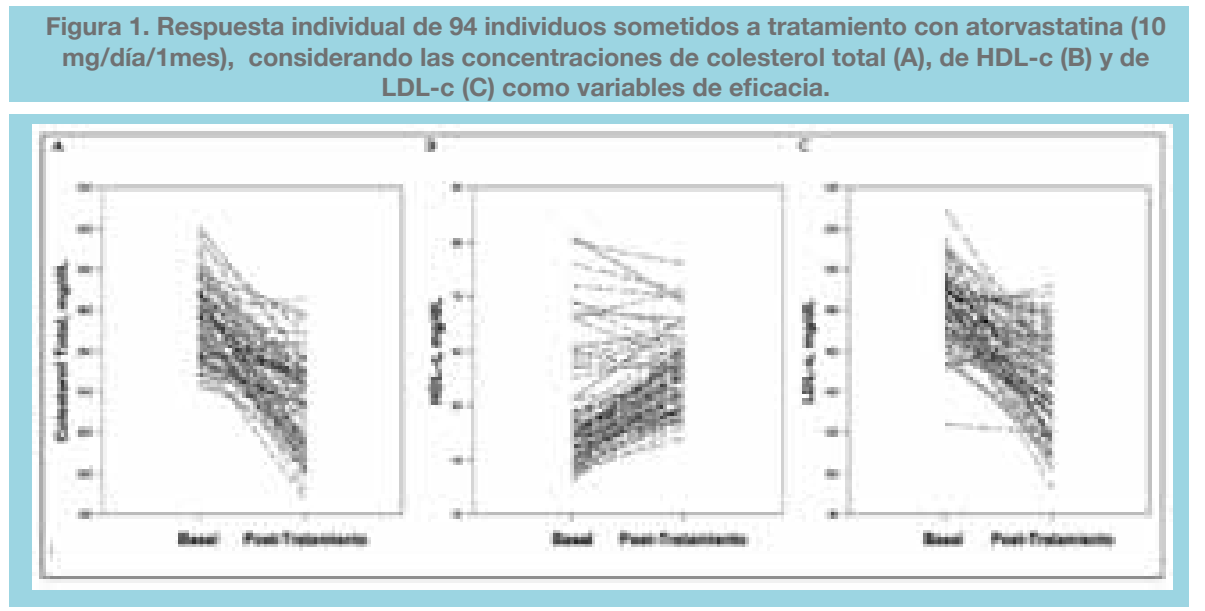

do nuevas hipótesis con respecto al funcionamiento de las enzimas asociadas al metabolismo y transporte de los fármacos, como también a la etiología de la enfermedad cardiovascular. Por ello, en este estudio, hemos analizado, por primera vez en nuestro país, el efecto de tres polimorfismos presentes en los genes CYP3A4 $(-290 \mathrm{~A}>\mathrm{G})$ y $\mathrm{ABCB} 1(3435 \mathrm{C}>\mathrm{T}$ y $2677 \mathrm{G}>\mathrm{A} / \mathrm{T})$ sobre la respuesta al tratamiento con atorvastatina $(10 \mathrm{mg} /$ día/1 mes).

El análisis de los datos bioquímicos, demuestra que atorvastatina ( $10 \mathrm{mg} / \mathrm{dí} / 1 \mathrm{mes})$ es eficaz en reducir los niveles séricos de LDL-c (-20\% en promedio), principal variable de eficacia. Además, redujo significativamente las concentraciones séricas de colesterol total $(-14 \%)$ y triglicéridos $(-23 \%)$, y aumentó significativamente los niveles de HDL-c (+19). Estos datos permiten también confirmar la variabilidad de respuesta a este fármaco (Figura 1).

Desde el punto de vista de distribución genotípica y frecuencias alélicas, nuestros datos demuestran que para el polimorfismo $3435 \mathrm{C}>\mathrm{T}$ del exón 21 del gen $\mathrm{ABCB} 1$, la frecuencia en nuestra población para el alelo salvaje $\mathrm{C}$ fue 0.686 ; siendo similar a la frecuencia reportada por Wielandt et al..$^{29}$ en etnias mapuche y mestiza de nuestro país, observando frecuencias alélicas de 0.650 y 0.670 , respectivamente, para este alelo. Similarmente, Rodrígues et al. ${ }^{26}$ reportaron una frecuencia de 0.530 para el alelo $C$ en población brasileña hipercolesterolémica. Además, la frecuencia encontra$\mathrm{da}$, en nuestro estudio, para el alelo $\mathrm{C}$ de la variante $3435 \mathrm{C}>\mathrm{T}$ es similar a la observada en población asiática $(0.631)^{30}$, pero difiere significativamente de la observada en poblaciones de origen caucásico $(0.480)^{25}$, ${ }^{31}$; africano $(0.830)^{31}$, oceánico $(0.47)^{32}$, afroamericano $(0.790)^{10}$ o centroamericano $(0.510-0.540)^{33}$.
Con respecto al alelo silvestre $\mathrm{G}$ del polimorfismo 2677G $>A / T$ del gen ABCB 1, la frecuencia alélica encontrada fue 0.590 , levemente inferior a la reportada en poblaciones étnicas chilenas $(0.650-0.690)^{29}$. Al comparar nuestros resultados con otras poblaciones, podemos observar una similitud con las reportadas por Crettol et al..$^{34} \mathrm{y}$ Cascorbi et al..$^{25}$, quienes observaron en poblaciones caucásicas frecuencias alélicas de 0.55 y 0.56 , respectivamente. En contraste, Yi et al ${ }^{30}$ reportaron, en un estudio con 232 individuos de nacionalidad coreana, una frecuencia alélica de 0.440 para este alelo. Por su parte, Rodrigues et al. ${ }^{26}$, en población brasileña, reportaron una frecuencia de 0.580 para el alelo $2677 \mathrm{G}$, similar a la observada en nuestro estudio (0.590).

En cuanto al polimorfismo -290A $>$ G del gen CYP3A4, el alelo mutado $\mathrm{G}$ se encontró en el 11,2\% de los individuos estudiados. Al comparar esta frecuencia (0.112) con la reportada por otros estudios, podemos observar una similitud con población brasileña $(0.133)^{27}$, asiática $(0.160)^{10}$ e hispánica $(0.093)^{35}$, y una diferencia significativa con población americana, tanto de raza blanca $(0.360)$ como negra $(0.540)^{35}$.

En relación al efecto de los polimorfismos investigados sobre la respuesta terapéutica a atorvastatina, un hallazgo importante en nuestra investigación, fue la relación observada entre la presencia del alelo mutado -290G del gen CYP3A4 y la mejor respuesta terapéutica a atorvastatina en los individuos portadores de esta variante. Se observó una mayor reducción de los niveles de colesterol total y LDL-c, al concluir la terapia farmacológica, en los individuos portadores de este alelo. Además, estos individuos presentaron un mayor aumento de los niveles de HDL-colesterol posttratamiento. Este resultado es similar al encontrado por 
Gao et al..$^{36}$, en población asiática, quienes observaron que los portadores del alelo mutado $\mathrm{G}$ presentaban una mayor reducción de los niveles séricos de colesterol total, al ser tratados con atorvastatina $20 \mathrm{mg} /$ día por 4 semanas. Sin embargo, nuestros datos no coinciden con los resultados obtenidos por Kajinami et al. ${ }^{16}$, quienes estudiaron 340 sujetos hipercolesterolémicos tratatos por 52 semanas con $10 \mathrm{mg} /$ día de atorvastatina. Estos autores observaron que la presencia de la variante $\mathrm{G}$ se asocia a niveles más elevados de LDLcolesterol post-tratamiento.

Por otro lado, nuestros resultados no mostraron efecto de los polimorfismos $3435 \mathrm{C}>\mathrm{T}$ y $2677 \mathrm{G}>\mathrm{A} / \mathrm{T}$ del gen ABCB1 sobre la respuesta terapéutica a atorvastatina. Similarmente, Rodrigues et al. ${ }^{26}$, en población brasileña, no observaron una relación entre la presencia individual de estos polimorfismos y la respuesta a atorvastatina (10 mg/día/1mes). Sin embargo, estos autores consiguieron establecer una asociación entre la reducción de niveles lipídicos de colesterol total y LDL-c, al realizar un análisis de haplotipos, construidos con los alelos de estas dos variantes del gen ABCB1.

En resumen, los resultados obtenidos en nuestro estudio sugieren que el polimorfismo -290A $>$ G del gen CYP3A4 es capaz de modificar la respuesta terapéuti- ca a la atorvastatina (10mg/día/1mes) en la población estudiada. Como es conocido, las estatinas son el fármaco de elección para el tratamiento de la hipercolesterolemia, no sólo por sus excelentes resultados en la disminución de los niveles lipídicos, sino también por la disminución del riesgo cardiovascular. Otro aspecto importante a considerar, es que esta terapia farmacológica es distribuida en todos los servicios de atención médica pública de nuestro país, razón por la cual el conocimiento de las principales rutas del metabolismo de este medicamento es de suma importancia y podría ser un factor indispensable en la decisión del clínico en la correcta elección del tratamiento farmacológico y la dosis a utilizar. Resulta interesante investigar, en un futuro estudio, si este polimorfismo puede influenciar la respuesta a otras dosis de atorvastatina, y también a otras estatinas disponibles en nuestro país. También es importante considerar que este estudio debe ser replicado por otros grupos de investigación, idealmente con un mayor número de sujetos, con el objetivo de evaluar un posible efecto dosis alélica-respuesta, situación que no fue posible estudiar en nuestra población por la ausencia de individuos portadores del genotipo homocigoto GG. 


\section{Referencias:}

1. LANAS F, AVEZUM A, BAUTISTA LE, DÍAZ R, LUNA M, ISLAM S, et al. Risk factors for acute myocardial infarction in Latin America: the INTERHEART Latin American study. Circulation 2007; 115: 1067-74.

2. MINISTERIO DE SALUD. GOBIERNO DE CHILE. Encuesta Nacional de Salud. 2003

3. MEDINA E, KAEMPFFER A. Epidemiology of cardiovascular disease in Chile. Rev Chil Cardiol 2007; 26: 219 - 26.

4. NEWMAN CB, PALMER G, SILBERSHATZ H, SZAREK M. Safety of atorvastatin derived from analysis of 44 completed trials in 9,416 patients. Am J Cardiol 2003; 92: 692-5

5. VAUGHAN CJ, GOTTO AM, BASSON CT. The evolving role of statins in the management of atherosclerosis. J Am Coll Cardiol 2000; 35: 1-10.

6. MARHUENDA E. Estatinas en el tratamiento de dislipidemias. Ars. Pharmacautica 2002; 43: 83-85

7. ECHEVERRI D, BUITRAGO L, MONTES FR. Efecto pleiotrópico de las estatinas características farmacológicas útiles en la prevención tratamiento y regresión de la enfermedad cardiovascular. Rev Col Cardiol 2005; 12:103-112.

8. LIAO JK. Beyond lipid lowering: the role of statins in vascular protection. Int J Cardiol. 2002; 86: 5-18.

9. NAVARRO-LÓPEZ. F. Bases genéticas de la enfermedad coronaria. Rev Esp Cardiol 2002; 55: 413-31.

10. THOMPSON JF, MAN M, JOHNSON KJ, WOOD LS, LIRA ME, LLOYD DB, et al. An association study of 43 SNPs in 16 candidate genes with atorvastatin response. Pharmacogenomics Journal 2005; 5: 352-58.

11. NEUVONEN PJ, NIEMI M, BACKMAN JT. Drug interactions with lipid-lowering drug: mechanisms and clinical relevance. Clin Pharmacol Ther. 2006; 80: 565-81.

12. SALAZAR LA, HIRATA MH, QUINTÃO EC, HIRATA RDC. Lipid-lowering response of the HMG-CoA reductase inhibitor fluvastatin is influenced by polymorphisms in the low-density lipoprotein receptor gene in Brazilian patients with primary hypercholesterolemia. J Clin Lab Anal 2000; 14: 125-31.

13. KAJINAMI K, AKAO H, POLISECKI E, SCHAEFER EJ. Pharmacogenomics of statin responsiveness. Am J Cardiol. 2005; 96:65K-70K

14. WANG A, YU BN, LUO CH, TAN ZR, ZHOU G, WANG
LS, et al. Ile118Val genetic polymorphism of CYP3A4and its effects on lipid-lowering efficacy of simvastatin in Chinese hyperlipidemic patients. Eur J Clin Pharmacol 2005; 60:843848.

15. KEROLA T, LEHTIMÄKI, KÄHÖNEN M, NIEMINEN T. Statin pharmacogenomics: lipid response and cardiovascular outcomes. Curr Cardio Risk Rep 2010; 4: 150-158.

16. KAJINAMI $\mathrm{K}$, BROUSSEAU ME, ORDOVAS JM, SCHAEFER EJ. CYP3A4 genotypes and plasma lipoprotein levels before and after treatment with atorvastatin in primary hypercholesterolemia. Am J Cardiol 2004; 93:104-7.

17. SCHMITZ G, LANGMANN T. Pharmacogenomics of cholesterol-lowering therapy. Vascul Pharmacol. 2006; 44:75-89.

18. MEGA JL, MORROW DA, BROWN A, CANNON CP, SABATINE MS. Identification of genetic variants associated with response to statin therapy. Arteroscler Thromb Vas Biol 2009; 29:1310-1315.

19. EXECUTIVE SUMMARY OF THE THIRD REPORT OF THE NATIONAL CHOLESTEROL EDUCATION PROGRAM (NCEP) Expert Panel on Detection, Evaluation, and Treatment of High Blood Cholesterol in Adults (Adult Treatment Panel III). JAMA. 2001; 285: 2486-97.

20. FOSSATI P, MEDICCI R. Abstract Book. International Symposium on Cholesterol Control and Cardiovascular Diseases: Prevention and Therapy. Milan, Italy, 1987. Apud: Bayer Corporation, Diagnostic Division, Tarrytown, N.Y., CholesterolFast color.

21. FOSSATI P, PRINCIPE L. Serum triglycerides determined colorimetrically with an enzyme of low-density lipoprotein cholesterol in plasma without use of the preparative ultracentrifuge. Clin Chem 1982; 28:2077 - 80.

22. BURSTEIN M, SCHOLNICK HR, MORFIN R. Rapid method for the isolation of lipoproteins from human serum by precipitation with polyanions. J Lipid Res 1970; 11: 583 - 95.

23. FRIEDEWALD WT, LEVY RI, FREDICKSON DS. Estimation of the concentration of low-density lipoprotein cholesterol in plasma, without use of the preparative ultracentrifuge. Clin Chem 1972; 18:499 - 502.

24. SALAZAR LA, HIRATA MH, CAVALLI SA, MACHADO MO, HIRATA RD. Optimized procedure DNA isolation from fresh and cryopreserved clotted human blood useful in clinical molecular testing. Clin Chem 1998; 44:1748-50.

25. CASCORBI I, GERLOFF T, JOHNE A, MEISEL C, HOFFMEYER S, SCHWAB M, et al. Frequency of single nucleotide 
polymorphisms in the P-glycoprotein drug transporter MDR 1 gene in white subjects. Clin Pharmacol Ther 2001; 69:169-74.

26. RODRIGUES AC, REBECCHI IM, BERTOLAMI MC, FALUDI AA, HIRATA MH, HIRATA RDC. High baseline serum total and LDL cholesterol levels are associated with MDR1 haplotypes in Brazilian hypercholesterolemic individuals of European descent. Braz J Med Biol Res 2005; 38: 1389-97.

27. CAVALLI SA, HIRATA MH, HIRATA RDC. Detection of MboII polymorphism at the $5^{\prime}$ promoter region of CYP3A4. Clin Chem 2001; 47: 348-351.

28. ROMERO T, ROMERO CX. Prevención cardiovascular estancada: tendencias alarmantes y barreras socioeconómicas persistentes. Rev Esp Cardiol 2010; 63:1340-8.

29. WIELANDT AM, VOLLRATH V, CHIANALE J. Polimorfismos del gen de Resistencia a múltiples drogas (MDR1) en poblaciones chilenas: mapuche, mestiza y maorí. Rev Méd Chile 2004; 132:1061-1068.

30. YI SY, HONG KS, LIM HS, CHUNG JY, OH DS, KIM JR, et al. A variant 2677A allele of the MDR1 gene affects fexofenadine disposition. Clin Pharmacol The. 2004; 76:418-27.

31. AMEYAW MM, REGATEIRO F, LI T, LIU X, TARIQ M, MOBAREK A, et al. MDR1 pharmacogenetics: frequency of the C3435T mutation in exon 26 is significantly influenced by ethnicity. Pharmacogenetics. 2001;11:217-221

32. ROBERTS RL, JOYCE PR, MULDER RT, BEGG EJ, KENNEDY MA. A common P-glycoprotein polymorphism is associated with nortriptyline-induced postural hypotension in patients treated for major depression. Pharmacogenomics J. 2002; 2:191-6

33. VICENTE J, SINUES B, FANLO A, VASQUEZ P, MEDINA JC, MARTINEZ-JARRETA B. Polymorphism C3435T of the MDR1 gene in Central Americans and Spaniards. Mol Biol Rep. 2008; 35:473-8.

34. CRETTOL S, DÉGLON JJ, BESSON J, CROQUETTEKROKAR M, HÄMMIG R, GOTHUEY I, et al. ABCB1 and cytochrome $\mathrm{P} 450$ genotypes and phenotypes: Influence on methadone plasma levels and response to treatment. Clin Pharmacol Ther 2006; 80: 668-81.

35. BALL S, SCATINA J, KAO J, FERRON G, FRUNCILLO $\mathrm{R}$, MAYER $\mathrm{P}$, et al Population distribution and effects on drug metabolism of a genetic variant in the 5 , promotor region of CYP3A4. Clin Pharmacol Ther. 1999 ;66:288-94

36. GAO Y, ZHANG L, QIANG F. CYP3A4*1G polymorphism is associated with lipid-lowering efficacy of atorvastatin but not of simvastatin. Eur J Clin Pharmacol. 2008 64:877-882. 